\title{
CRISES, RÉVOLTES ET OCCASION RÉVOLUTIONNAIRE CHEZ MARX ET LÉNINE
}

Par Irene VIPARELLI

Esquisser une analyse du lien entre crises et révoltes sociales dans le marxisme s'avère une tâche plus ou moins problématique. Il suffit de s'attarder quelques instants sur les manières dont Marx lui-même aborde le problème des crises capitalistes pour comprendre que l'on s'engage dans une recherche qui semble lui être étrangère. En effet, lorsque la crise est vue comme un "pur " phénomène économique, le problème de l'action de forces subjectives n'est absolument pas posé; lorsque, au contraire, la crise est abordée dans sa signification plus étendue, c'est-à-dire en tant que " crise révolutionnaire ", où sont impliquées les dimensions politique et idéologique, le discours politique porte plutôt sur la question de savoir comment profiter des "occasions révolutionnaires " qu'elle offre. Dans l'une et l'autre perspective, la question des révoltes sociales paraît tout à fait absente. Face à un tel silence théorique, comment s'orienter?

Il nous semble que deux possibilités sont ouvertes: soit on se borne au cercle étroit des textes où une révolte particulière fait l'objet d'une analyse spécifique, soit, comme aurait dit Althusser, on interroge le "silence " marxien, en cherchant à poser la question "implicite » du lien entre crise et révoltes sociales à partir de la question « explicite » du lien entre crise du capitalisme et " occasions révolutionnaires ${ }^{1}$. En suivant la première approche, on raterait, au moins partiellement, notre objet. En effet, si l'on se focalisait, par exemple, sur les premières révoltes ouvrières, survenues lors du passage de la manufacture à la grande industrie, qui font l'objet des analyses marxiennes dans l'article sur la révolte des tisseurs en Silésie de 1843 et des pages célèbres du chapitre XV du Capital, il serait impossible d'aborder la question de la relation entre révoltes prolétaires et crises du capitalisme arrivé à maturité. Il nous semble donc préférable de suivre la seconde des voies envisagées, en se lançant à la recherche de l' " objet invisible »- les révoltes sociales -, que l'on supposera caché entre 
les lignes des analyses plus générales relatives au lien entre crise et occasion révolutionnaire. Il nous faudra donc préciser au préalable ce qu’il faut entendre, d'une part, par crise et par conjoncture révolutionnaire et, d'autre part, par « révolte sociale " et par occasion révolutionnaire. Nous nous tournerons ensuite vers les analyses du lien entre crise et conjoncture révolutionnaire chez Marx et Lénine.

Pourquoi se concentrer sur les analyses de Marx et Lénine, alors que la question des révoltes sociales a acquis une centralité bien plus majeure chez d'autres auteurs, comme Rosa Luxemburg ou le Gramsci de "L'Ordine Nuovo ", et dans d'autres courants du marxisme, tels que l' "Operaismo » italien des années 1960 ? Paradoxalement, parce que la question des révoltes n'occupe pas chez Marx et Lénine une place centrale. C'est précisément parce que les révoltes sociales constituent chez eux un "objet invisible ", qu'il faut chercher à l'intérieur de la problématique plus générale de la relation entre crises du capitalisme et conjonctures révolutionnaires, qu'ils offrent deux perspectives absolument privilégiées, et non convergentes, pour cerner la spécificité marxiste du lien entre crise et révoltes sociales.

\section{CRISE ET " OCCASION RÉVOLUTIONNAIRE ". QUEL RÔLE POUR LES RÉVOLTES SOCIALES?}

Que faut-il entendre par "révolte sociale »? Les coordonnées de la réponse nous sont données par Marx dans les pages du chapitre xv du Capital consacrées aux premières révoltes ouvrières. On y trouve en effet évoquées des caractéristiques qui, loin de concerner seulement les révoltes prolétaires, constituent plutôt les racines de toute révolte sociale. La première est annoncée par Marx dans sa description des révoltes menées par les ouvriers contre les machines: "Sous sa forme-machine [...] le moyen de travail devient immédiatement le concurrent du travailleur. Le rendement du capital est dès lors en raison directe du nombre d'ouvriers dont la machine anéantit les conditions d'existence $»^{2}$. Les révoltes sont toujours, en premier lieu, une conséquence du désespoir causé par l'aggravation des conditions d'existence. La deuxième caractéristique est mise en lumière par la définition marxienne des grèves comme " révoltes périodiques du travail contre l'autocratie du capital $»^{3}$. Les révoltes sont ainsi liées à des sentiments de haine et de ressentiment envers les formes politico-juridiques de l'exploitation capitaliste. Enfin, le dernier élément constitutif de toute révolte est explicité par Marx lorsqu'il déclare: «Il faut du temps et de l'expérience avant que les ouvriers, ayant appris à distinguer entre la machine et son emploi capitaliste, dirigent leurs atta- 
ques non contre le moyen matériel de production, mais contre son mode social d'exploitation $»^{4}$. Le lien entre le phénomène de la révolte et la " confusion-fausseté " quant aux moyens et aux buts de la lutte, c'est-àdire l'idéologie, s'avère tout à fait décisif pour une "définition marxiste " de la " révolte ". Le terme " révolte " acquiert ainsi chez Marx une double signification, désignant à la fois la "révolte-spontanéité ", c’est-à-dire les formes de la lutte de classes qui sont dominées par les sentiments de désespoir et de ressentiment, et la " révolte-idéologie ", à savoir les moments de la lutte de classes qui sont dominés par une conception illusoire des moyens et des buts à atteindre. En un mot, le terme " révolte » désigne les phases de la lutte de classes où les sentiments et l'idéologie priment sur les formes conscientes et révolutionnaires de lutte.

Mais quel rôle les révoltes jouent-elles à l'intérieur des conjonctures révolutionnaires ouvertes par les crises capitalistes? Pour répondre à cette question, il serait absolument inapproprié de ne considérer les crises que comme de "purs " phénomènes économiques. Pour conceptualiser le lien entre crise et "action subjective ", il faut que la crise soit prise en charge dans sa signification plus "étendue ", c'est-à-dire en tant que crise sociale où sont impliquées les dimensions idéologique et politique. Il nous faudra ici garder à l'esprit les enseignements d'Althusser sur le caractère surdéterminé des contradictions capitalistes, sur la détermination en "dernière instance " par l'économie, et enfin sur le " déplacement " de la contradiction principale dans les périodes de crise $^{5}$. Tout cela nous apprend, tout d'abord, qu'il n'y a aucune raison pour qu'une crise économique produise naturellement une révolte ${ }^{6}$; ensuite, qu'une conjoncture révolutionnaire, loin d'être seulement engendrée par le facteur économique, peut être ouverte aussi bien par une crise économique, lorsque ses effets se propagent dans la dimension politique et idéologique (comme ce fut le cas dans les conjonctures de 1848 et de 1905), que par une crise où la dimension soit politique soit idéologique est dominante et où, par conséquent, l'élément économique ne constitue pas la cause immédiatement déterminante. Ce fut le cas, par exemple, des conjonctures de 1870 ou de 1917 , ouvertes par des crises politiques consécutives aux défaites militaires.

On parvient ainsi à définir la " conjoncture révolutionnaire " comme l'« espace dialectique » situé entre les deux « situations limites » de l'écla-

5. Voir L. Althusser, Pour Marx, Paris, Maspero, 1965. II sera bien utile de nous souvenir aussi de l'affirmation de Gramsci dans le Cahier 13: " On peut exclure que, par elles-mêmes, les crises économiques immédiates produisent des événements fondamentaux. [...] La question particulière du malaise ou du bien-être économique comme cause de réalités historiques nouvelles est un aspect partiel de la question des rapports de forces dans leurs différents degrés. [...] On peut donc dire que tous ces éléments sont la manifestation concrète des fluctuations conjoncturelles de l'ensemble des rapports de forces sociaux, sur le terrain desquels se produit le passage de ces rapports-là aux rapports de forces politiques, pour culminer dans le rapport militaire décisif ". (A. Gramsci, Cahiers de prison (10-13), trad. fr. P. Fulchignoni, G. Granel et N. Negri, Paris, Gallimard, 1978, pp. 384-385.) 
tement d'une crise " purement " économique, qui ne provoque aucune révolte sociale, et d'une "crise révolutionnaire ", où toute une série de conditions - à la fois économiques, politiques, idéologiques, nationales et internationales - et leurs imbrications réciproques imposent la transformation de la crise en révolution. En somme, la conjoncture révolutionnaire est le contexte où il faut que toute une série de conditions à la fois objectives et subjectives s'imposent afin que la crise puisse se transformer en occasion révolutionnaire.

Portant sur les révoltes sociales, c'est-à-dire sur une forme de la lutte de classes, notre analyse devra cependant se borner aux conditions subjectives. Par conséquent, les deux "situations limites " de notre champ d'analyse seront constituées, d'une part, par la " pure révolte », où aucune des conditions subjectives pour profiter de l'occasion révolutionnaire n' est encore réalisée, et, d'autre part, par l' "insurrection comme art ", où toutes les conditions subjectives sont désormais réalisées ${ }^{7}$. À l'intérieur d'un tel champ d'analyse, il s'agira d'éclaircir ce qu'il faut entendre à la fois par "révolte spontanée " et par "révolte-idéologie", tout en montrant comment l'une et l'autre favorisent ou empêchent la formation d'une subjectivité capable de profiter des occasions révolutionnaires ${ }^{8}$.

La "révolte spontanéité " constitue la forme la plus immédiate de réaction subjective à la crise (si réaction il y a). Les classes exploitées, aussi bien la paysannerie que la petite bourgeoisie et le prolétariat, peuvent être amenées à la révolte par le désespoir dû à l'aggravation de leurs conditions matérielles et par le ressentiment provoqué par la radicalisation de la contradiction capitaliste entre production de richesse et pauvreté sociale. Étant donné le caractère spontané et immédiat de cette forme de lutte, excluant toute organisation, chaque classe commence alors par être poussée à se révolter de façon isolée, pour "soi-même"; mais les sentiments de révolte agissent aussi comme un facteur de cohésion et d'agrégation sociale qui permet aux classes de dépasser leur défiance réciproque en vue d'une lutte commune.

Ainsi, même si cette phase de la lutte ne laisse pas envisager une « occasion révolutionnaire ", elle tend à créer une "masse populaire » unifiée qui constitue la conditio sine qua non de toute révolution. Autrement dit, le désespoir et la haine contre la misère et l'exploitation, en poussant chaque classe à la révolte et en concourant à la formation d'une « union sentimentale » entre les classes, représentent la première forme de subjectivation des masses et, par conséquent, la " préhistoire » de tout mouvement révolu-

7. Voir V. I. Lénine, "Le marxisme et l'insurrection", in V. I. Lénine, Fuvres, tome XXVI, Paris, Éditions sociales, 1958, pp. 13-19.

8. Une telle analyse, faisant abstraction de l'imbrication réciproque des deux significations du terme révolte, ne pourra que pécher par schématisme. Mais notre objectif est seulement de restituer les coordonnées générales à l'intérieur desquelles Marx et Lénine pensent le lien entre crise, révolte et occasion révolutionnaire. 
tionnaire. C'est pour cette raison qu'aussi bien Marx que Lénine donnent toujours à ces formes spontanées de lutte une signification positive: pour Marx, elles représentent l'« école " de la révolution; quant à Lénine, il les considère comme les formes « embryonnaires » de la révolution.

La « révolte-idéologie » désigne le moment de la domination de la petite bourgeoisie dans le champ des forces révolutionnaires: à cause de sa propre position moyenne entre la bourgeoisie et le prolétariat dans les rapports de production capitalistes, elle est tout à fait incapable d'atteindre un point de vue autonome de classe. Par conséquent, sa conscience s'avère composée d'un mélange contradictoire de principes libéraux bourgeois et de principes socialistes prolétaires, donnant lieu à des constructions théoriques où la valeur et les fins de la révolte sont considérées dans le cadre d'une séparation, tout à fait unilatérale, abstraite et dogmatique, entre les deux dimensions économique et politique.

Dans sa "version " irréalisable, l'idéologie petite-bourgeoise coïncide avec la perspective anarchique: la séparation entre la dimension économique et politique est accomplie à travers l'identification immédiate de la "dimension politique " et de la "dimension étatique " de l'" oppression sociale ». Par conséquent, d'une part, le moment " politique » de la lutte de classes, celui de la conquête du pouvoir d'État, est nié; d'autre part, la révolution, contrainte dans le domaine de la seule lutte économique, est conçue comme une "grève générale " qui, simultanément, détruit la dimension politique, tout en réalisant l'émancipation sociale. Ainsi, les anarchistes souhaitent réaliser les buts révolutionnaires de la lutte, c'està-dire l'abolition de l'État et de l'exploitation, sans accepter pourtant les moyens nécessaires pour les atteindre. C'est à cette condition seulement que la révolte sociale peut être le dernier mot de la lutte des classes.

Dans sa «version » incohérente, l'idéologie petite-bourgeoise est un " opportunisme ", où la séparation mécanique entre les dimensions économique et politique se réalise grâce à l'autonomisation des deux " organes » de la lutte prolétaire. Le syndicat constitue l'instrument de la lutte « réformiste " pour l'amélioration progressive des conditions matérielles du prolétariat - bien plus que comme un instrument de politisation des révoltes sociales, il est l'instrument de leur canalisation; le Parti, quant à lui, est engagé dans le travail de civilisation, d'éducation et de formation d'une conscience politique des masses - son action politique est tout à fait détachée de la dynamique des révoltes sociales. Cette «opposition opportuniste " partage le point de vue anarchiste, qui conçoit les " grèves " comme une forme " purement économique » de lutte, et elle réduit la dimension de la lutte politique au cercle trop étroit de la seule lutte parlementaire. Il s'agit simplement d'éduquer les masses grâce aux libertés 
formelles dont on dispose dans le système représentatif bourgeois, dans l'attente que le capitalisme affirme les conditions objectives de son propre dépassement ${ }^{9}$. Ainsi la lutte parlementaire, qui devrait être un instrument de la lutte de classes, cesse de l'être.

Il est intéressant de mentionner la manière dont Rosa Luxemburg réévalue, dans Grève de masse, parti et syndicat, l'action spontanée des masses, tout en critiquant ces deux points de vue. En effet, l'histoire des " grèves " en Russie depuis 1896 et l'utilisation des " grèves de masse " pendant la révolution de 1905 falsifient l'un et l'autre. D'une part, le développement des révoltes sociales sous forme de grèves constitue un instrument d'éducation des masses bien plus puissant que le parlementarisme: "Aussi une année de révolution a-t-elle donné au prolétariat russe 'l'éducation' que trente ans de lutte parlementaire et syndicale ne peuvent artificiellement donner au prolétariat d'Allemagne ${ }^{10}$. D'autre part, la " grève » est loin d'être une forme de lutte "purement économique »: "L'élément économique et l'élément politique, dans la période de grèves en masse, bien éloigné de se distinguer nettement ou même de s'exclure comme le veut le pédantisme schématique, constituent, au contraire, que deux faces entremêlées de la lutte de classe prolétarienne en Russie ${ }^{11}$. Alors que la lutte syndicale et la lutte parlementaire constituent des formes "réformistes" de lutte lorsqu'elles sont autonomisées, leur union dialectique dans la praxis les transforme en moyens de la lutte révolutionnaire ${ }^{12}$.

Il faut donc en conclure que la domination de la " révolte-idéologie " représente le moment où la révolte se transforme en une force négative et réactionnaire, qui désagrège et affaiblit le champ révolutionnaire. L'absence d'une stratégie de lutte vraiment révolutionnaire dans le cas de l'anarchie, la circonscription de la lutte au domaine trop étroit de la lutte parlementaire dans le cas de l'opportunisme, empêchent les masses de profiter des occasions qui leur sont offertes par la crise, transformant ainsi la révolte spontanée des masses en une nouvelle passivité qui les laisse impuissantes et désarmées face à l'initiative réactionnaire de la bourgeoisie.

"De toutes les classes qui, à l'heure actuelle, s'opposent à la bourgeoisie, seul le prolétariat est une classe vraiment révolutionnaire ${ }^{13}$. Le prolétariat seul, à cause de sa position dans les rapports de production bourgeois, peut opposer au libéralisme bourgeois son propre point de vue de classe indépendant et une vision cohérente des moyens à utiliser en vue des buts politiques à atteindre. Par conséquent, l'affirmation de l'hégémonie du pro-

9. C'est notamment la perspective dominante dans la Deuxième Internationale, défendue aussi bien par le « dernier Engels » que par Kautsky et qui fait l'objet de la critique à la fois de Lénine et de R. Luxemburg. 
létariat sur la petite bourgeoisie et sur la paysannerie représente la forme de subjectivité révolutionnaire arrivée à maturité, où les sentiments de révolte sont désormais soumis aux objectifs révolutionnaires de la lutte.

On peut maintenant, pour conclure cette partie générale, essayer de formuler une définition du rôle joué par la révolte dans les conjonctures révolutionnaires. D'une part, elle constitue la première figure, "spontanée ", de subjectivisation des masses, et donc le début de toute révolution; d'autre part, elle est marquée par une « confusion idéologique » quant aux moyens et aux buts de la lutte, et peut donc constituer une force réactionnaire. En raison de cette double signification, elle représente le moment proprement dialectique de chaque conjoncture révolutionnaire. Lorsque les sentiments de révolte sont soumis aux buts révolutionnaires, les conditions subjectives de la transformation de la crise en occasion révolutionnaire sont réalisées; lorsqu'au contraire, la dimension de la "révolte-idéologie » est dominante, la crise ne pourra que trouver une solution contre-révolutionnaire.

\section{CRISE, RÉVOLTE ET OCCASION RÉVOLUTIONNAIRE CHEZ MARX}

La définition par Marx des révoltes comme "école des masses " évoque une analogie avec la relation "adolescence-maturité ", qui nous indique que le passage de la révolte à la révolution est à concevoir comme un processus d'auto-émancipation. L'expérience concrète de la lutte de classe, c'est-à-dire la "praxis révolutionnaire ", donne aux masses à la fois une expérience progressivement plus mûre de leurs propres conditions d'existence et une conscience toujours plus claire des conditions de leur propre émancipation. "Révolte-spontanéité " et "révolte-idéologie " représentent pour Marx deux phases d'apprentissage des masses et, par conséquent, deux étapes successives dans le procès au cours duquel la subjectivité révolutionnaire peut se former.

Dans un tel dispositif, quel rôle faut-il attribuer aux crises, comme celle, économique, de 1848, ou celle, politique, engendrée par la guerre franco-allemande en 1870 ? Les périodes de crise correspondent aux moments historiques où aussi bien la démystification des rapports sociaux que la faiblesse politique des classes dominantes permettent à la lutte de classes de se développer dans ses formes les plus radicales. Constituant les lieux privilégiés de l'action subjective, les conjonctures révolutionnaires représentent donc le moment de vérification du degré de maturité acquis par les masses et le contexte historique de leur apprentissage ultérieur. En d'autres termes, les conjonctures révolutionnaires constituent le contexte où la "praxis révolutionnaire " fait accomplir aux masses, " en pratique ", le dépassement aussi bien de la phase de la "révolte-spontanéité » que 
de la "révolte-idéologie ", en leur permettant d'atteindre une forme de subjectivité révolutionnaire plus mûre.

Mais il importe d'écarter toute ambiguïté quant à une possible interprétation « évolutionniste » du dispositif marxien. Il est vrai qu'on vient de décrire un procès tout à fait linéaire où, en partant de la désorganisation de masse, on parvient, au moyen de la révolte, à la constitution de la subjectivité révolutionnaire. Cette linéarité doit toutefois être interprétée comme une tendance historique, qu'il ne faut pas confondre avec une conception téléologique du processus historique. C'est en fait la dimension conjoncturelle qui préserve le dispositif marxien de tout idéalisme: chaque conjoncture révolutionnaire, en constituant le lieu privilégié de l'action subjective, implique des formes de lutte de classes tout à fait nouvelles et originales qu'il est impossible de saisir a priori. Par conséquent, la dimension créative et éducative de la révolte, liée à cette dimension conjoncturelle et événementielle de l'histoire, loin de représenter une phase de la lutte s'effaçant lors de la maturation de la subjectivité révolutionnaire, constitue plutôt un élément inéliminable et perpétuel de la lutte de classes. Le moment historique du passage de la révolte à la révolution doit être considéré, lui aussi, comme une "tendance ", c'est-à-dire comme l'augmentation progressive des possibilités subjectives de saisir les occasions révolutionnaires, sans pourtant jamais constituer une réalisation pleine et entière des conditions subjectives de la victoire révolutionnaire.

\section{LE DISPOSITIF MARXIEN " EN ACTION ». \\ ENTRE LA CONJONCTURE DE 1848 ET CELLE DE 1871.}

Entre la révolution de février 1848 et la Commune de Paris, toutes les formes de la lutte de classes ont été expérimentées par les classes françaises, des révoltes spontanées à la révolution. Une analyse du déroulement des événements survenus pendant ces deux conjonctures nous permettra donc de montrer " en pratique » le procès de maturation de la subjectivité révolutionnaire, capable de saisir les occasions révolutionnaires qui lui sont offertes par la crise.

La crise économique qui éclate en Angleterre pendant les années 1845-1847 ouvre une conjoncture révolutionnaire dans l'Europe tout entière $^{14}$. En France, elle amène aussi bien à la révolution bourgeoise de février 1848 qu'à l'inauguration d'un procès d'intensification de la lutte de classes: l'aggravation de la misère par la crise, d'un côté, le ressentiment provoqué par la politique du gouvernement bourgeois, de l'autre, poussent chacune des classes exploitées, à la fois le prolétariat, la petite bourgeoisie et la paysannerie, à se révolter. Ainsi, grâce à cette réaction 
tout à fait spontanée à la crise, chaque classe fait son entrée comme sujet actif de l'histoire, inaugurant son procès de subjectivation.

En juin 1848, le prolétariat fut conduit à se révolter: "Les ouvriers n'avaient plus le choix : il leur fallait mourir de faim ou engager le combat. [...] C'est la bourgeoisie qui força le prolétariat de Paris à s'insurger en juin ${ }^{15}$. La politique anti-prolétaire menée par la bourgeoisie, se concrétisant dans la lutte contre les Ateliers Nationaux, avait contribué à durcir les conditions d'existence déjà désespérées du prolétariat et avait radicalisé le ressentiment prolétaire, anéantissant son illusion de "pouvoir s'émanciper aux côtés de la bourgeoisie ${ }^{16}$. Cependant, comme toute révolte, l'insurrection de juin fut une "école »: "C'est seulement après avoir été trempé dans le sang des insurgés de Juin que le drapeau tricolore est devenu le drapeau de la révolution européenne, le drapeau rouge ${ }^{17}$.

"Le 10 décembre 1848, ce fut le jour de l'insurrection paysanne ${ }^{18}$ : la politique fiscale de la bourgeoisie, contribuant à aggraver la misère endémique de la paysannerie française, avait poussé celle-ci à la révolte. Louis Bonaparte fut à la fois le représentant du désespoir et de la haine paysanne envers la "République des riches" ${ }^{19}$ et l'incarnation de leurs illusions quant à la tradition bonapartiste. Cependant, cette " révolte électorale " fut, elle aussi, une école: seule l'action politique concrète de Bonaparte aurait pu libérer " une partie des paysans français de l'illusion napoléonienne [...]. Le processus se déroula sous la forme d'une lutte incessante entre les maîtres d'école et les prêtres ${ }^{20}$.

Enfin, face à la récusation des Concordats à l'amiable et aux tendances contre-révolutionnaires de la bourgeoisie française, la petite bourgeoisie s'insurge, le 19 juin 1849, à la manière petite-bourgeoise. Elle aurait voulu mener une manifestation pacifique en défense de la Constitution, pourtant « le cortège [...] fut reçu sur les boulevards d'une façon très peu parlementaire par les dragons et les chasseurs de Changarnier ${ }^{21}$. Mais, grâce à cette défaite, la petite bourgeoisie prit conscience de sa propre impuissance de classe et de la nécessité de s'allier avec le prolétariat.

L'expérience pratique des défaites apprend aux classes exploitées l'exigence de s'allier contre la bourgeoisie et donc d'avancer vers des formes plus mûres de la lutte de classe. Ainsi, pendant la dernière période de la conjoncture révolutionnaire de 1848, une nouvelle phase du processus de subjectivation des masses est inaugurée: « Le 10 mars était une révolution.

15. K. Marx, Les luttes de classes en France, in K. Marx, Euvres IV. Politique I, présentée et annotée par M. Rubel, Paris, Gallimard, 1994, pp. 259-261. 
Derrière les bulletins de vote, il y a les pavés ${ }^{22}$. Les élections du 10 mars 1850 représentaient pour Marx une véritable « occasion révolutionnaire »: d'une part, toutes les classes exploitées étaient désormais alliées dans le parti social-démocrate, d'autre part, la bourgeoisie était affaiblie, divisée et fragilisée par la guerre contre Bonaparte. Il aurait donc fallu considérer la victoire électorale comme le début d'un procès de radicalisation de la lutte de classe aboutissant à la révolution. Pourtant, " la signification révolutionnaire du 10 mars, la réhabilitation de l'insurrection de Juin, fut enfin complètement anéantie par la candidature d'Eugène Sue $»^{23}$. Le parti social-démocrate, dominé par la petite bourgeoisie, au lieu de considérer la victoire électorale comme un moyen en vue de la révolution, l'avait au contraire interprétée comme " un but en soi »; l'occasion révolutionnaire s'était évanouie et la réaction bourgeoise avait triomphé.

Pourtant, cette domination de la "révolte idéologie " constitue, elle aussi, un moment d'apprentissage nécessaire des masses: "jusqu'au 2 décembre, [la révolution] avait accompli la moitié de ses préparatifs, et elle

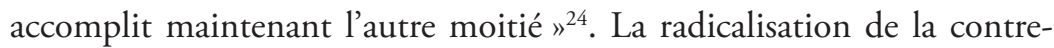
révolution constitue à la fois le résultat de l'attitude réformiste de la petite bourgeoisie et l'instrument pour démontrer " en pratique " l'impossibilité de toute solution conciliatrice. Elle s'avère donc être le moyen d'anéantir la domination idéologique petite-bourgeoise et d'affirmer l'hégémonie de la perspective révolutionnaire de l'avant-garde prolétaire.

La conjoncture révolutionnaire de 1871, ouverte par la crise politico-sociale consécutive à la défaite de la France dans la guerre francoallemande, représente le moment de vérification de la maturité acquise par la subjectivité révolutionnaire grâce à l'expérience de 1848: d'une part, le prolétariat s'est désormais affirmé comme classe d'avant-garde des forces révolutionnaires; d'autre part, les autres classes exploitées, au moyen de l'école de 1848 et du Second Empire, ont dû accepter l'hégémonie prolétaire: "Le paysan était bonapartiste, parce que la grande Révolution, avec tous les bénéfices qu'il en avait tirés, se personnifiait à ses yeux en Napoléon. Cette illusion, qui se dissipa rapidement sous le second Empire [...], ce préjugé du passé, comment aurait-il résisté à l'appel de la Commune aux intérêts vivants et aux besoins pressants de la paysannerie? "25. Quant à la petite bourgeoisie, économiquement et politiquement ruinée par le Second Empire, elle ne pouvait que garder une attitude neutre face à la Commune, qui « l'avait sauvée, en réglant sagement cette cause perpétuelle de différends à l'intérieur même de la 
classe moyenne: la question des créanciers et débiteurs $»^{26}$.

L'attitude des masses face à la crise se révèle, elle aussi, tout à fait opposée à celle de 1848. En 1848, la crise permet à la bourgeoisie de profiter des sentiments de désespoir et de ressentiment du prolétariat, contraint à la révolte, pour le massacrer; en mars 1871, bien au contraire, c'est le prolétariat qui profite de la faiblesse politico-économique de la bourgeoisie pour lui soustraire l'initiative historique. Face aux tentatives bourgeoises de résoudre la crise par le biais de la capitulation, le prolétariat transforme la crise en " occasion révolutionnaire ", s’engageant à la résoudre à sa manière. Grâce à la transformation positive de la crise en révolution, l'avant-garde prolétaire soumet les sentiments des révoltes des classes exploitées aux buts révolutionnaires et accomplit ainsi "dans la praxis» le passage à la forme la plus mûre de la lutte de classes: la révolution.

\section{CRISE, RÉVOLTE ET OCCASION RÉVOLUTIONNAIRE CHEZ LÉNINE}

L'expérience révolutionnaire de 1905 est conçue par Lénine comme l'une des conditions préalables de la victoire révolutionnaire de 1917. Il semblerait donc tout à fait convenable de poser la question du lien entre crise et révoltes sociales chez Lénine en utilisant la même méthode que celle adoptée pour l'analyse de Marx. Une comparaison entre les deux conjonctures révolutionnaires, dont la première constitue l'« école " préparant les masses à la seconde, permettrait d'analyser les différentes étapes à accomplir dans le procès de maturation d'une subjectivité révolutionnaire. Cependant, une rapide comparaison entre la conjoncture de 1848 et celle de 1905 suffit à démontrer qu'une telle approche théorique visant à établir une continuité, à la fois historique et méthodologique, entre Marx et Lénine impliquerait l'effacement de l'objet de notre analyse.

En effet, 1905 fut la conséquence aussi bien de la crise industrielle et commerciale de 1902 que de la guerre russo-japonaise qui s'ensuivit. Face à cette crise économique et politique du régime tsariste, Lénine envisage deux possibilités historiques: soit le peuple, profitant de l'« occasion historique " qui lui est offerte par la crise, prend la tête de la révolution et impose la « voie révolutionnaire " pour l'affirmation du capitalisme et, partant, des conditions objectives de son propre dépassement; soit la bourgeoisie, s'emparant de l'initiative historique, impose la "voie réformiste " grâce à toutes sortes de compromis entre la grande bourgeoisie et les classes réactionnaires ${ }^{27}$.

On voit donc que, loin de s'intéresser à la conjoncture en tant que 
dominée par la dimension de la révolte, comme Marx dans le cas de 1848 et Rosa Luxemburg dans ses propres analyses de 1905, Lénine pose le problème de savoir comment saisir l'" occasion révolutionnaire ». Si l'on suit le schéma marxien, dans lequel la révolte et la révolution sont conçues comme deux étapes successives d'un même procès, il faut en conclure que la question des " révoltes sociales » ne se pose pas chez Lénine, puisque le moment de la révolte est toujours déjà périmé par l'urgence du problème révolutionnaire. Ou, plutôt, elle se pose de façon seulement négative, le concept de révolte renvoyant soit à la spontanéité populaire indisciplinée et violente, celle d'une violence sociale désordonnée en réponse à l'arbitraire de la répression, qui doit être autant que possible disciplinée ${ }^{28}$, soit à la valorisation romantique et petite-bourgeoise de la révolte ${ }^{29}$.

Toutefois, lorsqu'il désigne les Soviets comme la forme la plus avancée d'organisation spontanée des masses, c'est-à-dire comme la troisième étape dans le processus de constitution de la subjectivité révolutionnaire, après juin 1848 et la Commune de Paris, Lénine paraît utiliser exactement le dispositif marxien de l'évolution progressive de la révolte à la révolution par le moyen de la praxis révolutionnaire. Quelle est la valeur attribuée par Lénine à un tel développement "pratique " de la subjectivité révolutionnaire? Considère-t-il, lui aussi, qu'en engageant les masses dans la praxis révolutionnaire, la révolte elle-même a la capacité d'enclencher le passage de la révolte à la révolution? Ou s'agit-il plutôt d'un dispositif théorique différent où la question des révoltes sociales est posée en d'autres termes?

Abordons ces questions à partir de l'analyse par Lénine du rôle des Soviets dans les conjonctures révolutionnaires. Une double possibilité est envisagée: soit ils se constituent comme organes du pouvoir populaire, soit une "mauvaise infinité » les transforme en organes du système de la domination bourgeoise. Une double possibilité qui, en 1917, revêtait la forme d'un choix dramatique entre " tout le pouvoir aux Soviets » et la défaite de la révolution ${ }^{30}$. "Les Soviets, nous dit Lénine, ne peuvent se développer véritablement, ne peuvent pleinement accomplir leurs tâches et déployer leurs possibilités que s'ils prennent tout le pouvoir d'État, autrement ils nont rien à faire, autrement ils ne sont que des embryons (et il n'est pas possible d'être longtemps un embryon), ou des jouets ${ }^{31}$. On voit que Lénine aborde ici le problème du rapport entre maturation des forces révolutionnaires et révolution d'une manière opposée à celle de Marx. Alors que, pour ce dernier, la révolution est l'effet de la maturité 
acquise par les forces révolutionnaires, pour Lénine, au contraire, la révolution, c'est-à-dire la conquête du pouvoir politique, est ce qui permet cette maturation. Il semble bien évident qu'on est en présence d'un dispositif théorique tout à fait différent. La question du passage des révoltes à la révolution, loin d'être dépassée, y est reformulée d'une façon tout à fait originale. Au couple "école-maturité ", qui tendait à concevoir le passage de la révolte à la révolution comme un procès d'auto-transformation et d'auto-émancipation (par le biais de la praxis des masses), Lénine oppose le couple "embryon-organisme ", où le procès d'organisation n'apparaît plus comme une auto-transformation, mais comme l'action de « donner une forme " (par le biais du parti d'avant-garde et d'une théorie, voire du pouvoir d'État) à ce qui apparaît comme une figure indéterminée et ambiguë (la révolte). Pour le dire autrement, "révolte-spontanéité " et "révolution" ne sont plus conçues comme deux étapes différentes d'un même processus, mais plutôt comme deux figures qualitativement différentes de la lutte de classes: la révolte constitue la dimension de l'organisation spontanée des masses, qui demeure toujours dans l'ambiguïté de la double évolution possible en force révolutionnaire ou en organe de la domination bourgeoise. Le problème n'est plus celui du processus qui conduit de la révolte vers la révolution; l'enjeu, chez Lénine, est plutôt le "saut qualitatif " à accomplir du niveau spontané de la lutte de classes à son niveau révolutionnaire ${ }^{32}$. Il s'agit de changer le terrain de la lutte.

Quelle est la force capable de réaliser ce "saut qualitatif »? La réponse léniniste se trouve dans la définition de la relation dialectique entre le Parti d'avant-garde et les masses, résolvant ainsi le problème du rapport entre théorie et praxis d'une manière tout à fait opposée à Marx. Alors que, pour Marx, c'est la pratique révolutionnaire qui représente la force décisive dans le procès de constitution de la subjectivité et de la théorie révolutionnaire, pour Lénine, au contraire, «sans théorie révolutionnaire, pas de mouvement révolutionnaire $»^{33}$.

Dans la théorie de Lénine, "révolte-spontanéité ", "révolte-idéologie » et " révolution " représentent les trois «moments " constitutifs de l'histoire en général et de chaque « tournant révolutionnaire » en particulier, la « révolte-idéologie » et la " théorie » constituant les deux attitudes opposées de la pensée face à la «vie » spontanée des masses. Il faut donc préciser, d'une part, quelle signification acquièrent ces termes dans le dispositif de Lénine, et, d'autre part, ce qui est en jeu dans cette imbrication entre la vie spontanée des masses, l'idéologie et la théorie.

La dimension de la "révolte-spontanéité " constitue le terrain où 
l'on expérimente toutes les formes de lutte: de la lutte terroriste jusqu'à l'insurrection des masses, de la lutte parlementaire jusqu'au boycottage, des grèves économiques jusqu'aux grèves politiques, des révoltes violentes des paysans jusqu'à l'appropriation des terres par la paysannerie pauvre, des manifestations pacifiques jusqu’à la lutte armée. Quelle que soit la forme acquise par la lutte, cette dimension est toujours dominée soit par les sentiments de révolte, soit par toutes sortes d'idéologies. Dans le domaine de la lutte spontanée, le prolétariat, loin de pouvoir conquérir un quelconque rôle d'avant-garde, reste incapable de s'élever au-dessus de la révolte, sa propre conscience immédiate de classe n'étant pas révolutionnaire mais réformiste, c'est-à-dire trade-unioniste.

À partir de cette dimension de la "vie ", c'est-à-dire des rapports de forces entre les classes donnés par la lutte de classes spontanée, la fonction de la théorie marxiste est d'indiquer aux masses le chemin à parcourir pour pousser la lutte "en avant ", au-delà de cette spontanéité. Il faut qu'en montrant aux masses, à chaque tournant historique, quelle est la tactique la plus adéquate aux buts politiques à atteindre, elle accomplisse le «saut » vers la lutte révolutionnaire. En d'autres termes, la théorie, en mettant en lumière, à chaque tournant de l'histoire, à la fois les contradictions historiques et la guerre civile qui y est représentée, doit s’imposer comme le "guide pour l'action » des masses ${ }^{34}$.

L'idéologie petite-bourgeoise, au contraire, est toujours « en retard " par rapport à la spontanéité des masses: au lieu de prendre la « vie » et les sentiments de révolte comme point de départ pour leur donner une conscience et une direction, au lieu de pousser "en avant " l'organisation spontanée des masses, elle se révèle tout aussi ignorante des contradictions historiques et de la lutte des classes qu'incapable de s'affirmer comme un " guide pour l'action ». En raison de son détachement de la vie, elle reste une " doctrine » abstraite et impuissante qui ne peut que se soumettre à la spontanéité, se bornant à ratifier a posteriori, à chaque tournant de l'histoire, les rapports de forces entre les classes donnés par la lutte spontanée.

\section{LE DISPOSITIF LÉNINISTE EN ACTION.}

\section{LA CONJONCTURE DE 1917}

L'attitude différente de la théorie et de l'idéologie face à la vie sociale se manifeste clairement dans les conjonctures révolutionnaires, lorsque la crise intensifie l'élan révolutionnaire du peuple en le poussant spontanément à la révolte. La théorie, soumettant l'élan des masses aux buts révolutionnaires, constitue la force permettant à la révolte d'accomplir le "saut » dans la révolution; l'idéologie, au contraire, apparaît comme une 
force réactionnaire qui, du fait de son incapacité à indiquer aux masses quels sont les moyens et les objectifs à atteindre, immobilise la lutte dans ses figures embryonnaires et indéterminées, ce qui implique désorganisation des masses, perte de l'occasion révolutionnaire et renversement de la révolution en contre-révolution.

Voyons comment ce dispositif se traduit dans une conjoncture historique donnée: celle, révolutionnaire, de 1917, engendrée par la crise politique qui éclate en Russie à la suite de la guerre impérialiste mondiale. L'analyse de la relation entre le parti bolchévique et les masses permet de mettre en lumière que l'idéologie ne représente pas simplement une attitude opposée à celle de la théorie, mais qu'elle constitue plutôt le danger le plus grand de la théorie elle-même: la relation dialectique entre le Parti et la masse risque toujours de se renverser dans la séparation idéologique entre une "doctrine abstraite de la révolution " et la révolte spontanée des masses. "Tous nos efforts", écrivait Lénine pendant la conjoncture révolutionnaire de 1917, «doivent tendre [...] à ne pas retarder sur les événements, de façon à pouvoir éclairer au mieux les ouvriers et les travailleurs au fur et à mesure, sur les modifications de la situation et de la lutte des classes. Car telle est en ce moment la tâche principale du parti: expliquer aux masses que la situation est extrêmement critique, que toute action peut se terminer par une explosion, et qu'un soulèvement prématuré pourrait, par conséquent, faire le plus grand mal $\aleph^{35}$.

Lors des tournants révolutionnaires, la tâche organisatrice du Parti s'avère bien difficile: le parti bolchévique risque de retarder sur la vitesse avec laquelle les rapports de forces entre les classes changent, ce qui impliquerait, d'une part, l'incompréhension du "tournant historique " en cours, l'incapacité à adopter une tactique adéquate et l'affirmation d'une attitude idéologique envers la «vie » des masses, et, d'autre part, le danger d'une explosion spontanée de l'élan révolutionnaire du peuple, la répression bourgeoise et la perte de l'« occasion révolutionnaire ".

Juillet 1917 représente le premier de ces « tournants brusques » où le parti bolchévique assume, sans le vouloir, une attitude idéologique: le massacre du prolétariat au début de juillet 1917 constituait l'épilogue d'une période de luttes spontanées du prolétariat, commencée les 20 et 21 avril et poursuivie le 18 juin, présentant toutes les caractéristiques des révoltes ${ }^{36}$. Quelle avait été l'attitude bolchévique face aux révoltes spontanées du prolétariat? «L'erreur réelle de notre parti, dans les journées des 3 et 4 juillet [...] a été seulement de considérer la situation générale comme moins révolutionnaire 
qu'elle ne l'était et de croire encore possible le développement pacifique des transformations politiques grâce à un changement de la politique des Soviets $»^{37}$. Alors qu'il aurait fallu formuler la tactique du Parti à partir de la "vie ", c'est-à-dire de la lutte de classes spontanée, les bolchéviques étaient restés liés au mot d'ordre du passage pacifique du pouvoir aux Soviets qui, nié « en pratique " par les révoltes prolétaires, était désormais devenu une doctrine vide et abstraite. Ainsi, au lieu d'organiser l'élan révolutionnaire des masses, les bolchéviques avaient contribué indirectement à la victoire de la contre-révolution bourgeoise.

En septembre, lors de la Conférence démocratique convoquée par les menchéviques et les socialistes révolutionnaires, les bolchéviques retardent à nouveau sur les événements historiques: "Plus on réfléchit au sens de la Conférence dite démocratique, plus on la considère avec attention de l'extérieur - et de l'extérieur, dit-on, on peut mieux voir plus se confirme la conviction que notre parti a commis une erreur en y participant. Il fallait la boycotter $»^{38}$. Le boycottage est pour Lénine la tactique à adopter quand les conditions sont mûres pour l'insurrection ${ }^{39}$; en septembre, pourtant, au lieu de profiter de la conjoncture favorable pour guider les masses vers la révolution, le parti bolchévique, en détournant l'attention des masses vers de faux objectifs tels que la "Conférence démocratique ", avait contribué à la perte de l'occasion révolutionnaire.

Enfin, en octobre, alors que toutes les conditions pour la victoire de l'insurrection sont réunies, le parti bolchévique risque, une fois de plus, de se renverser de guide des masses en force idéologique: une fraction du parti estimait ne pouvoir faire la révolution avant que le Congrès des Soviets lui ait donné la légitimité de la "majorité démocratique». Face à ce risque, écrit Lénine, "ma conviction la plus profonde est que, si nous 'attendons' le Congrès des Soviets et laissons tout de suite échapper l'occasion, nous causons la perte de la révolution ${ }^{40}$. Ne pas profiter de l'occasion révolutionnaire n'aurait eu d'autre signification que la trahison de la révolution, c'est-à-dire l'abandon de la « vie des masses » à la spontanéité, qui est en soi incapable d'accomplir « le saut » de la révolte à la révolution ${ }^{41}$. 